The following scientific article was officially published in the Proceedings of the 8th International Workshop on Systems, Signal Processing and their Applications (WoSSPA 2013), published by the IEEE. This article's citation is as follows:

Cheriet, Farida. "Multimodal image fusion of anatomical structures for diagnosis, therapy planning and assistance." In 8th International Workshop on Systems, Signal Processing and their Applications (WoSSPA), (2013): pp. 31-36.

doi: $\underline{10.1109 / \text { WoSSPA.2013.6602332 }}$

The manuscript, as accepted by the publisher, is reproduced in the following pages.

(C) 2013 IEEE. Personal use of this material is permitted. Permission from IEEE must be obtained for all other users, including reprinting/republishing this material for advertising or promotional purposes, creating new collective works for resale or redistribution to servers or lists, or reuse of any copyrighted components of this work in other works. 


\title{
MULTIMODAL IMAGE FUSION OF ANATOMICAL STRUCTURES FOR DIAGNOSIS, THERAPY PLANNING AND ASSISTANCE
}

\author{
Farida Cheriet ${ }^{a, b}$ \\ ${ }^{a}$ Department of Computer and Software Engineering, École Polytechnique de Montréal, Montréal, Qc, Canada \\ ${ }^{\mathrm{b}}$ Sainte-Justine University Hospital Research Center, Montréal, Qc, Canada
}

\begin{abstract}
This paper provides an overview of work done in recent years by our research group to fuse multimodal images of the trunk of patients with Adolescent Idiopathic Scoliosis (AIS) treated at Sainte-Justine University Hospital Center (CHU). We first describe our surface acquisition system and introduce a set of clinical measurements (indices) based on the trunk's external shape, to quantify its degree of asymmetry. We then describe our $3 D$ reconstruction system of the spine and rib cage from biplanar radiographs and present our methodology for multimodal fusion of MRI, X-ray and external surface images of the trunk. We finally present a physical model of the human trunk including bone and soft tissue for the simulation of the surgical outcome on the external trunk shape in AIS.
\end{abstract}

\section{INTRODUCTION}

Imaging systems of anatomical structures have known a wide evolution over the past decade and provide several measurable parameters with potential to quantify morphological and functional abnormalities. Interestingly, some imaging modalities provide functional content which is complementary to the structural content provided by other modalities. For instance, Magnetic Resonance Imaging (MRI) and single photon emission computed tomography (SPECT) are often used to assess myocardial viability, while multi-detector tomography (MDCT) and fluoroscopy are used to assess the coronary and ventricle morphology, even though a sequence of fluoroscopic images implicitly contains information about the contractility of the epicardium. The fusion of multimodal images of cardiac structures will thus allow a more accurate diagnosis of morphological and functional abnormalities, help in planning therapies and assist real time image-guided interventions.

In another clinical application, 3D assessment of the deformity of the spine in patients with scoliosis can be performed using stereo radiographic reconstruction techniques. These techniques provide a $3 \mathrm{D}$ model of the spine, according to which several 3D clinical parameters are computed and used for diagnosis, follow-up or correction assessment of the spinal deformity. A complete geometric model of the trunk can be obtained from multimodal fusion of MRI, X-ray and topographic images of the trunk. The geometric model can also be combined with mechanical properties of the bone structures and soft tissue to build a biomechanical model of the trunk that can be used, for example, in treatment simulations.

Scoliosis is a three-dimensional deformity of the spine and the rib cage, leading to a general asymmetry of the trunk. The most frequent type of scoliosis, idiopathic scoliosis, has no specific identifiable cause. The onset of the spinal deformation is variable and becomes more prominent during the adolescent growth spurt. Scoliosis is clinically apparent by observing the asymmetry of spinous processes, ribs, and scapulae, imbalance between the top and bottom of the spine, and left-right asymmetry of the trunk in forward bending [1]. Among the adolescent population, the incidence of adolescent idiopathic scoliosis (AIS) is $4.5 \%$. Among patients with AIS, $8 \%$ to $9 \%$ will be treated by brace and about 1 in 1,000 will need surgery using spinal instrumentation and fusion to correct the deformity. In the absence of treatment, it may lead to postural problems, and even cardiac or pulmonary complications.

Usually, the evaluation of scoliosis relies on frontal and lateral radiographs, on which several 2D measurements are computed. Most importantly, the Cobb angle, defined as the angle between the end plates of the two most tilted vertebrae along the spinal curve, represents a scoliosis severity index when measured in the frontal plane. Nevertheless, in the past fifty years, many studies have documented the three-dimensional aspect of scoliosis and emphasized the importance of a 3D evaluation [2].

X-rays only reveal the spinal deformity, while the trunk surface asymmetry associated with AIS has been shown to be the most important factor affecting the patient's mental and social well-being [3, 4]. A metaanalysis of the literature has demonstrated that the 
correction of the apparent deformity in idiopathic scoliosis was a major factor of satisfaction for patients who have had an operation [5]. Therefore, it would be useful to have an objective quantitative tool to evaluate and document the three-dimensional postoperative correction of the trunk external asymmetry. In this perspective, several methods to quantify scoliosis deformity from back or trunk surface asymmetry have been introduced since the 1970s [3, 6-11]. Our research team has for several years utilized non-invasive surface topography to acquire the whole trunk of patients at the Sainte-Justine University Hospital Center (CHU) scoliosis clinic in Montréal, Canada [12]. Several trunk shape indices have been developed and their reliability evaluated on patients in standing position [13].

The purpose of this paper is to provide an overview of ongoing research carried out by our group to simulate and document post-operative trunk surface shape in AIS patients having undergone spinal surgery at CHU SainteJustine. This work exploits a non-invasive surface digitizing system from Creaform Inc. to scan the pre- and post-operative trunk shapes of patients during their visits to the hospital's orthopedic clinic.

\section{TRUNK SURFACE ACQUISITION}

A non-invasive system allowing the acquisition and reconstruction of the 3D trunk surface geometry of spinal deformity patients was installed and has been in routine use at CHU Sainte-Justine since 2002. The system is located in a closed room in the orthopedics clinic wing, and is comprised of four serial digitizers (Capturor II LF, Creaform Inc.) [14] as seen in Figure 1. The four digitizers are arranged in such a way as to provide maximal coverage of the trunk surface. In sequence, the different units capture the back, the front, the left side and the right side of the patient. The two lateral units are angled obliquely (approx. $50^{\circ}$ on either side of the front unit's line of sight) and are placed slightly lower than the front and rear units, in order to minimize obstruction by the patient's arms. Each digitizer is fixed to a vertical support which allows for height adjustment. All four units are placed at a given standoff distance $(1500 \pm 100 \mathrm{~mm})$ from the center of the digitizing space, at which the patient must stand.

Each digitizer consists of a halogen structured light projector (white light source and grating slide) combined with a standard color CCD camera of resolution $1024 \mathrm{x}$ 768 pixels. A single acquisition comprises five images: four with phase-shifted fringes for measuring the geometry (using the interferometry principle combined with active triangulation) and one without fringes to get the surface texture data. The acquisition time for each digitizer is slightly over one second, and the total time for all four units to shoot in sequence is around five seconds. A software package provided with the digitizing system, FAPS, is used to control the acquisition process. It is then used to process the raw camera images to obtain a phase function, then to unwrap the phase function and convert

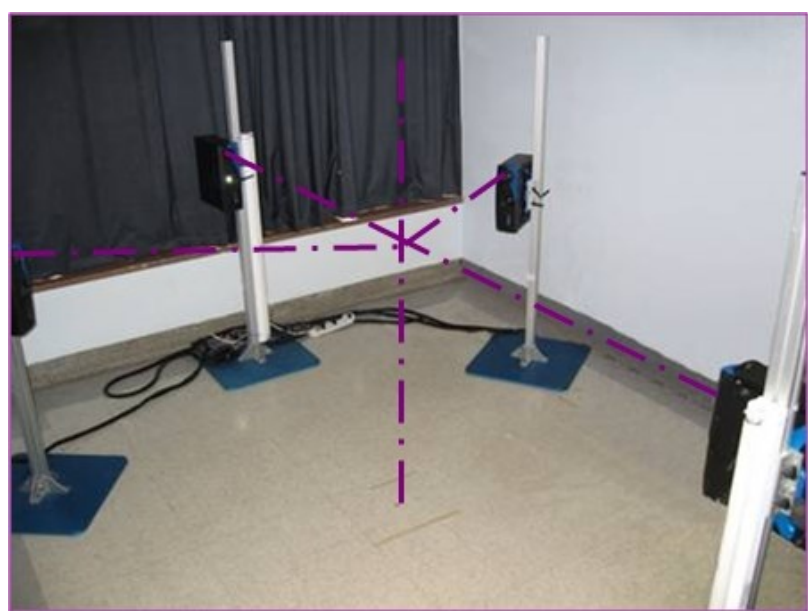

Figure 1. Clinical setup of four 3D optical digitizers (Creaform Inc.).

the resulting depth map into a metrical surface with texture mapped. Figure 2 illustrates this process in the case of the back of an anthropomorphic manikin.

To obtain the geometry of the entire trunk, the partial surfaces acquired by the four digitizers must be registered and merged. Here, a second software package also provided with the system, EM, is used to import the partial surfaces produced in FAPS, register them into a common reference frame and merge the geometries and textures into a single model. In order to register the partial surfaces properly, a multi-head calibration, exploiting a known planar reference target visible to all the cameras, is performed on a regular basis. The reconstructed surface region does not include the arms or the head. The resulting surface is a textured, high-density polygonal mesh. For an entire trunk of average size, the reconstructed polygonal mesh numbers around 180000 vertices. For easier manipulations, the data is usually subsampled with the result counting, depending of the patient's height, from 40000 to 70000 points. The EM program allows the complete model to be exported in a variety of file formats to facilitate subsequent analysis with our team's own in-house tools.

We have proposed a novel index to quantify trunk deformities associated with AIS from the external 3D surface of the trunk [15]. Thanks to its original functional representation using B-splines, this new index allows a smooth and local description of the deformation at all trunk levels and not only at the apex of the deformity. It thus provides a global description of the external trunk shape. The proposed index includes complementary measurements taken in the three planes: the back surface axial rotation, the trunk deviation in the frontal plane and the trunk deviation in the sagittal plane. The reliability of this index has been demonstrated on a cohort of 32 patients with AIS. For each patient, the surface of the trunk was acquired twice with patient repositioning. The reliability study also helped define, for each trunk level, a minimum interval beyond which a difference between two trunk acquisitions is significant. 


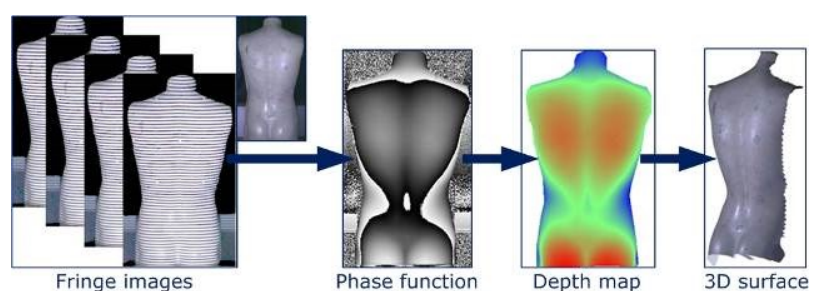

Figure 2. Steps to process the raw digitizer images (4 fringe +1 texture).

Furthermore, a cluster analysis conducted on a cohort of 236 individuals, including healthy adolescents and AIS patients, proved that the new index used to describe the trunk shape is also capable of distinguishing between healthy subjects and patients with scoliosis, and between different types of scoliotic trunk deformities according to the spinal curve type and its severity [16].

Moreover, a study conducted on 30 scoliotic patients showed that an Independent Component Analysis (ICA) parameterization of the trunk is able to capture local deformities such as asymmetries of the scapulae and shoulders [17].

\section{MULTIMODAL IMAGE FUSION}

The most challenging aspect in the fusion of multimodal images of the trunk is the deformation of the spine and the soft tissues between acquisitions in different patient postures: in our case, supine position during MRI acquisition and standing position during biplanar X-rays and trunk surface acquisitions, the latter two modalities being taken non-simultaneously. While many approaches were proposed for multimodal registration of images of deformable organs such as the heart [18], lungs [19], prostate [20] and kidneys [21], considerably less work has been done for multimodal registration of images of the trunk. Registration of spinal models has been addressed, but most of this work was aimed at assisted interventions where a $3 \mathrm{D}$ preoperative model of the spine obtained from $\mathrm{CT}$ or MR images is registered to $2 \mathrm{D}$ intra-operative images of the spine such as X-rays. A detailed review of 2D/3D registration techniques can be found in [22].

As the goal of the fusion of multimodal images of the trunk is the generation of a 3D full torso model including bone structures and soft tissue, the first step consists in the $3 \mathrm{D}$ reconstruction of the bone structures from multiplanar X-rays. To reconstruct the bone structures of the trunk, a calibrated system based on three digital multiplanar radiographs (postero-anterior view $\left(\mathrm{PA}-0^{\circ}\right)$, postero-anterior view with the $\mathrm{x}$-ray tube lifted up and angled down $20^{\circ}\left(\mathrm{PA}-20^{\circ}\right.$ ) and a lateral view (LAT) (see Figure 3) is employed [23]. We have also developed and validated novel techniques using digital multiplanar radiographs for $3 \mathrm{D}$ reconstruction and analysis of the

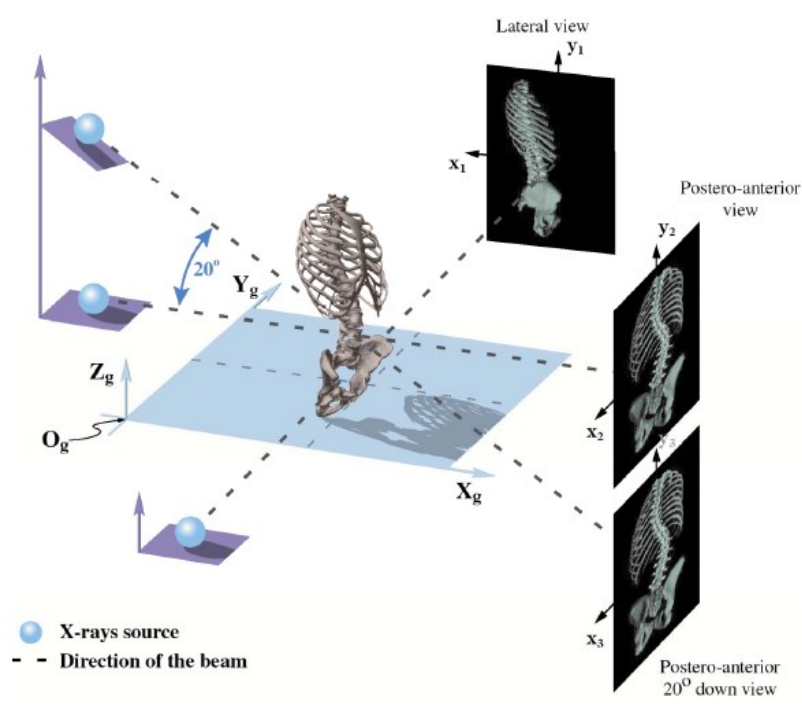

Figure 3. Stereo-radiography system for spine, pelvis and rib cage $3 \mathrm{D}$ reconstruction.

bone structures of the human trunk, including the spine, pelvis and ribcage [24-29].

The second step consists in fusing complementary information obtained from different modalities: soft tissues from MR images, 3D bone models from X-rays and the external surface of the trunk from 3D cameras. The key challenge is to establish a framework that will integrate the articulated model deformation of the spine [30-32] with an elastic deformation of the soft tissues to be able to fit the MR volume of the trunk, acquired in a different posture, within the space delimited by the external surface of the trunk and the surface of the underlying bone structures. We have proposed a closed form solution for the registration of MR images and of a 3D model of the spine reconstructed from biplanar radiography, using an articulated model [33]. It was shown that a completely rigid or completely elastic transformation was not able to compensate for the difference in posture between the two modality acquisitions. Finally, the soft tissue provided by the MR images was confined to the volume delimited by the trunk and bone surfaces using a constrained Thin-Plate Splines method [34].

Figure $4 \mathrm{a}$ shows a clear misalignment between the vertebrae extracted from X-ray data and those extracted from MRI data when rigid registration is used. The articulated model provides a better vertebral alignment but does not provide an adequate fitting between the MRI soft tissue data and the surface of the trunk (Figure $4 b$ ). The proposed method is able to compensate for the difference in posture during the acquisitions of the multimodal images of the trunk and yields a better alignment of the MRI and X-ray vertebrae geometries (Figure 4c). 


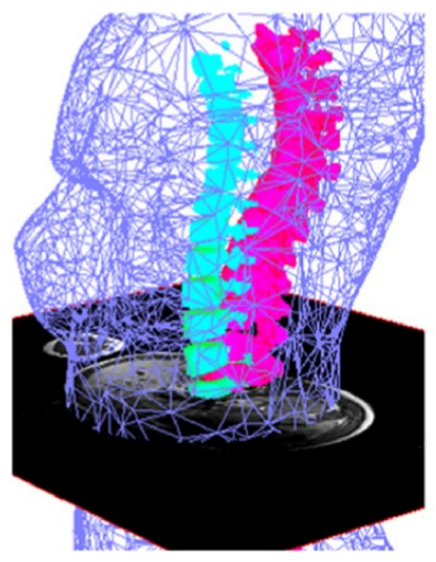

a) Rigid registration

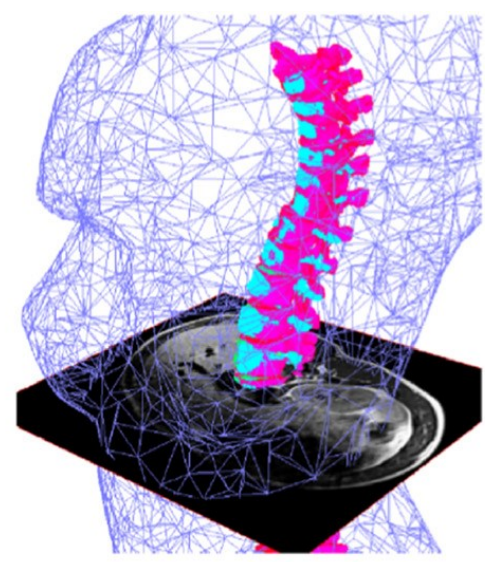

b) Articulated registration

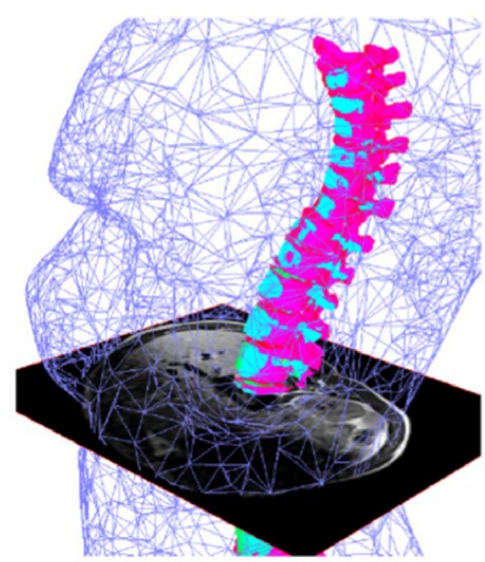

c) Proposed registration

Figure 4. Registration results comparing rigid registration with proposed method for the MRI, X-ray and surface topography (ST). X-ray vertebrae are displayed in pink, MRI vertebrae in cyan and ST (decimated mesh) in blue.

\section{PHYSICAL MODEL OF THE HUMAN TRUNK}

Spinal surgery for scoliosis consists in rectifying the spine shape using metal rods anchored to the vertebrae by means of screws and hooks. The prediction of surgical outcome is a fundamental element of any preoperative evaluation. Currently, the clinical method to define a surgical strategy and estimate the result of curve correction relies primarily on radiographic analysis of spinal flexibility and on the surgeon's own experience. To further assist the clinician during surgical planning, a biomechanical simulator is currently being developed at CHU Sainte-Justine to identify the optimal configuration of the implants to best correct the spinal deformities [35]. However, neither this simulator nor the spinal flexibility analysis consider the soft tissues of the trunk in order to provide information on the patient's expected external appearance after the intervention. For the surgeon, the predicted residual trunk asymmetry proves highly subjective and his experience remains his only asset. This is problematic considering that the main reason to prescribe an operation comes initially from the patient's dissatisfaction with their apparent deformity. At present, there is no tool available to estimate the effect of treatment on the patient's external appearance, even though surgeons' assessments of treatment outcomes are not significantly correlated with patient satisfaction [36].

Therefore, the goal of our work was to develop a simplified physical model of the deformable tissues between the skin surface (epidermis) and bone structures of the trunk in order to visualize in $3 \mathrm{D}$ and assess the effect of scoliosis surgery on the patient's external appearance [37] (see Figure 5).

\section{CONCLUSION}

The proposed approach for trunk shape analysis is complementary to the standard radiographic analysis used in surgery planning and evaluation of surgical outcome. Our methodology distinguishes itself by evaluating what is perceived by the patient as the major problem (asymmetrical appearance and rib hump) and what is considered by patients as the most important outcome, as opposed to what surgeons currently evaluate as the main outcome with Cobb angles and sagittal and coronal balance of the spine. By the same token, it could form the basis for recommendations on surgical strategies most likely to improve the patient's external appearance.

As for the surgical simulator based on a deformable model of the trunk, the potential benefits of this system will be as follows: 1) it will assist the surgeon, together with the patient, in deciding on the best surgical approach based on the resulting external trunk appearance; 2) it will provide an enhanced preoperative planning tool allowing the surgeon to take into account such factors as the residual external trunk asymmetry after the operation, when deciding on a surgical strategy including the indication for rib resection or anterior spine release; 3 ) it will allow the patient to readily understand the probable outcome as well as the risks of surgery and to be involved in the decisional process; 4) it has potential for generalized use by orthopedic surgeons.

\section{ACKNOWLEDGMENTS}

The author would like to thank Dr. Hubert Labelle and Dr. Stefan Parent who are collaborating orthopedic surgeons at CHU Sainte-Justine. The author is also grateful to Philippe Debanné and all the members of the LIV4D research group at École Polytechnique de Montréal for their contributions. This research was funded by the Natural Sciences and Engineering Research Council of Canada and the Canadian Institutes of Health Research. The equipment was financed by the Canadian Foundation for Innovation. 

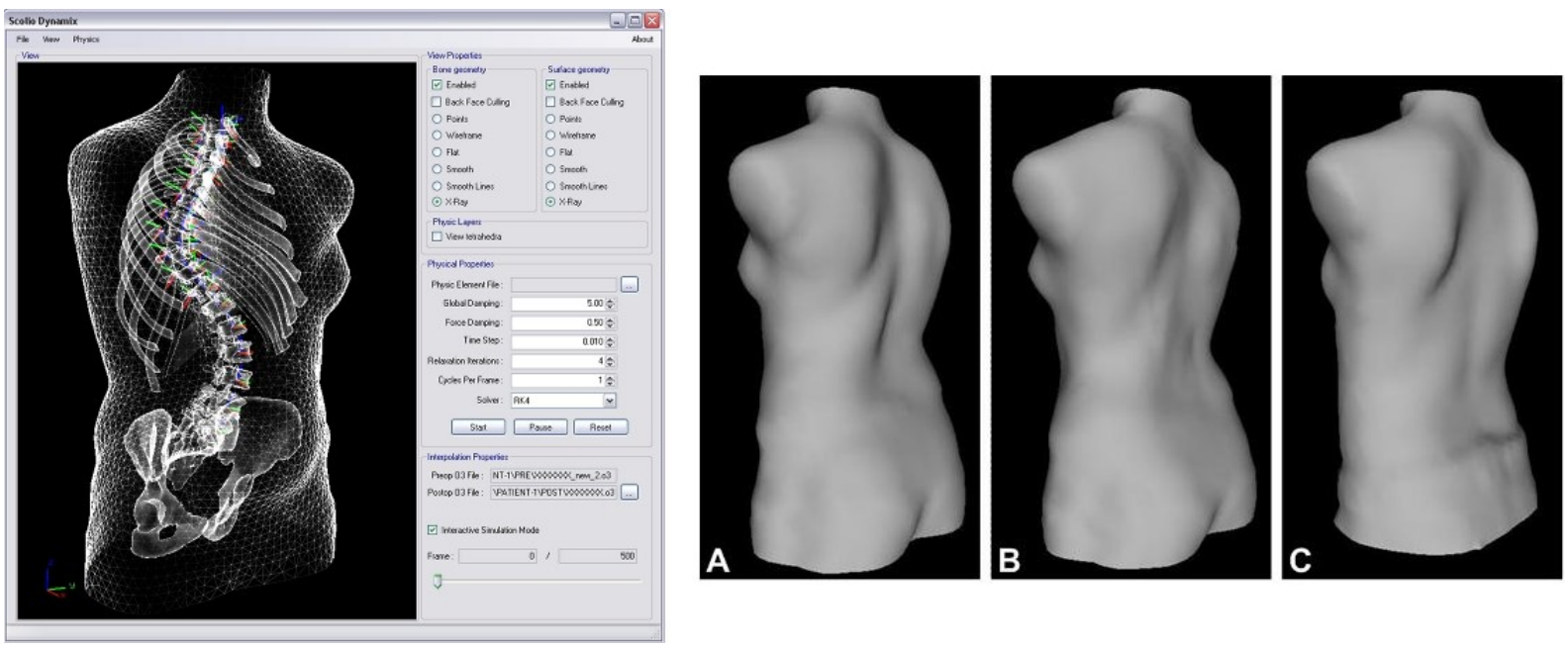

Figure 5. Left: Software GUI for controlling physical model simulation. Right: Sample result of a simulation on the external trunk shape: A) real pre-op trunk shape; B) simulated post-op trunk shape; C) real post-op trunk shape.

\section{REFERENCES}

[1] B.V. Reamy, and J.B. Slakey, "Adolescent idiopathic scoliosis: review and current concepts," American Family Physician, vol. 64, no. 1, pp. 111-116, 2001.

[2] H. Labelle, J. Dansereau, C. Bellefleur, and J.C. Jequier, "Variability of geometric measurements from three-dimensional reconstructions of scoliotic spines and rib cages," European Spine Journal, vol. 4, no. 2, pp. 88-94, 1995.

[3] C.J. Goldberg, M. Kaliszer, D.P. Moore, E.E. Fogarty, and F.E. Dowling, "Surface topography, Cobb angles, and cosmetic change in scoliosis," Spine, vol. 26, no. 4, pp. E55E63, 2001.

[4] R.K. Pratt, R.G. Burwell, A.A. Cole, and J.K. Webb, "Patient and Parental Perception of Adolescent Idiopathic Scoliosis Before and After Surgery in Comparison with Surface and Radiographic Measurements," Spine, vol. 27, no. 14, pp. 1543-1550, 2002.

[5] T.R. Haher, A. Merola, R.I. Zipnick, J. Gorup, D. Mannor, and J. Orchowski, "Meta-analysis of surgical outcome in adolescent idiopathic scoliosis. A 35-year English literature review of 11,000 patients," Spine, vol. 20, no. 14, pp. 15751584, 1995.

[6] M.S. Moreland, M.H. Pope, and D.G. Wilder, "Moire fringe photography of the human body," Medical Instrumentation, vol. 15, no. 2, pp. 129-132, 1981.

[7] I.A. Stokes, J. Armstrong, and M. Moreland, "Spinal deformity and back surface asymmetry in idiopathic scoliosis," Journal of Orthopaedic Research, vol. 6, no. 1, pp. 129-137, 1988.

[8] E.G. Dawson, M.A. Kropf, and G. Purcell, "Optoelectronic evaluation of trunk deformity in scoliosis," Spine, vol. 18, no. 3, pp. 326-331, 1993.
[9] T.N. Theologis, J.C.T. Fairbank, and A.R. Turner-Smith "Early Detection of Progression in Adolescent Idiopathic Scoliosis by Measurement of Changes in Back Shape With the Integrated Shape Imaging System Scanner," Spine, vol. 22, no. 11, pp. 1223-1227, 1997.

[10] J.G. Thometz, R. Lamdan, X.C. Liu, and L. R., "Relationship between Quantec measurement and Cobb angle in patients with idiopathic scoliosis," Journal of Pediatric Orthopaedics, vol. 20, no. 4, pp. 512-516, 2000.

[11] J.L. Jaremko, P. Poncet, J. Ronsky, J. Harder, J. Dansereau, H. Labelle, and R.F. Zernicke, "Indices of torso asymmetry related to spinal deformity in scoliosis," Clinical Biomechanics, vol. 17, no. 8, pp. 559-568, 2002.

[12] V. Pazos, F. Cheriet, L. Song, H. Labelle, and J. Dansereau, "Accuracy assessment of human trunk surface 3D reconstructions from an optical digitizing system," Medical and Biological Engineering and Computing, vol. 43, no. 1, pp. 1115, 2005.

[13] V. Pazos, F. Cheriet, J. Dansereau, J. Ronsky, R.F. Zernicke, and $\mathrm{H}$. Labelle, "Reliability of trunk shape measurements based on 3-D surface reconstructions," European Spine Journal, vol. 16, no. 11, pp. 1882-1891, 2007.

[14] L. Song, G. Lemelin, D. Beauchamp, S. Delisle, D. Jacques, and E.G. Hall, "3D measuring and modeling using digitized data acquired with color optical 3D digitizers and related applications," in Proc. 12th Symposium on $3 D$ Technology, Yokohama, Japan, Dec. 2001.

[15] L. Séoud, J. Dansereau, H. Labelle, and F. Cheriet, "Multilevel analysis of trunk surface measurements for noninvasive assessment of scoliosis deformities," Spine, vol. 37, no. 17, pp. E1045-E1053, 2012.

[16] L. Séoud, J. Dansereau, H. Labelle, and F. Cheriet, "Non Invasive Clinical Assessment of Trunk Deformities Associated with Scoliosis," IEEE Journal of Biomedical and Health Informatics, vol. 17, no. 2, pp. 392-401, 2013. 
[17] M. Adankon, N. Chihab, J. Dansereau, H. Labelle, and F. Cheriet, "Scoliosis Follow-Up Using Non-Invasive Trunk Surface Acquisition," IEEE Transactions on Biomedical Engineering, vol. PP, no. 99, p. 1, 2013.

[18] T. Mansi, X. Pennec, M. Sermesant, H. Delingette, and N. Ayache, "LogDemons Revisited: Consistent Regularisation and Incompressibility Constraint for Soft Tissue Tracking in Medical Images," in Medical Image Computing and ComputerAssisted Intervention (MICCAI 2010). vol. 6362, Springer, pp. 652-659, 2010.

[19] C.T. Metz, S. Klein, M. Schaap, T. van Walsum, and W.J. Niessen, "Nonrigid registration of dynamic medical imaging data using $\mathrm{nD}+\mathrm{t}$ B-splines and a groupwise optimization approach," Medical Image Analysis, vol. 15, no. 2, pp. 238-249, 2011.

[20] R. Alterovitz, K. Goldberg, J. Pouliot, I.C.J. Hsu, Y. Kim, S.M. Noworolski, and J. Kurhanewicz, "Registration of MR prostate images with biomechanical modeling and nonlinear parameter estimation," Medical Physics, vol. 33, no. 2, pp. 446454, 2006.

[21] J.L. Dillenseger, H. Guillaume, and J.J. Patard, "Spherical Harmonics Based Intrasubject 3-D Kidney Modeling/Registration Technique Applied on Partial Information," IEEE Transactions on Biomedical Engineering, vol. 53, no. 11, pp. 2185-2193, 2006.

[22] B. Fischl, M.I. Sereno, R.B.H. Tootell, and A.M. Dale, "High-resolution intersubject averaging and a coordinate system for the cortical surface," Human Brain Mapping, vol. 8, no. 4, pp. 272-284, 1999.

[23] F. Cheriet, C. Laporte, S. Kadoury, H. Labelle, and J. Dansereau, "A novel system for the 3-D reconstruction of the human spine and rib cage from biplanar X-ray images," IEEE Transactions on Biomedical Engineering, vol. 54, no. 7, pp. 1356-1358, 2007.

[24] S. Kadoury, F. Cheriet, C. Laporte, and H. Labelle, "A versatile $3 \mathrm{D}$ reconstruction system of the spine and pelvis for clinical assessment of spinal deformities," Medical and Biological Engineering and Computing, vol. 45, no. 6, pp. 591602, 2007.

[25] S. Kadoury, F. Cheriet, J. Dansereau, and H. Labelle, "Three-Dimensional Reconstruction of the Scoliotic Spine and Pelvis From Uncalibrated Biplanar x-Ray Images," Journal of Spinal Disorders and Techniques, vol. 20, no. 2, pp. 160-167, 2007.

[26] S. Kadoury, F. Cheriet, and H. Labelle, "Personalized XRay 3D Reconstruction of the Scoliotic Spine From Hybrid Statistical and Image-Based Models," IEEE Transactions on Medical Imaging, vol. 28, no. 9, pp. 1422-1435, 2009.

[27] S. Kadoury, F. Cheriet, M. Beausejour, I.A. Stokes, S. Parent, and H. Labelle, "A Three-Dimensional Retrospective Analysis of the Evolution of Spinal Instrumentation for the Correction of Adolescent Idiopathic Scoliosis," European Spine Journal, vol. 18, no. 1, pp. 23-37, 2009.
[28] S. Kadoury, F. Cheriet, and H. Labelle, "Self-Calibration of Biplanar Radiographic Imaging System Using Geometric Spine Shape Descriptors," IEEE Transactions on Biomedical Engineering, vol. 57, no. 7, pp. 1663-1675, 2010.

[29] F. Plourde, F. Cheriet, H. Labelle, and J. Dansereau, "Semiautomatic Detection of Scoliotic Rib Borders From Posteroanterior Chest Radiographs," IEEE Transactions on Biomedical Engineering, vol. 59, no. 4, pp. 909 - 919, 2012.

[30] J. Boisvert, F. Cheriet, X. Pennec, H. Labelle, and N. Ayache, "Articulated spine models for 3-D reconstruction from partial radiographic data," IEEE Transactions on Biomedical Engineering, vol. 55, no. 11, pp. 2565-2574, 2008.

[31] J. Boisvert, F. Cheriet, X. Pennec, H. Labelle, and N. Ayache, "Geometric variability of the scoliotic spine using statistics on articulated shape models," IEEE Transactions on Medical Imaging, vol. 27, no. 4, pp. 557-568, 2008.

[32] J. Boisvert, F. Cheriet, X. Pennec, H. Labelle, and N. Ayache, "Principal deformations modes of articulated models for the analysis of 3D spine deformities," Electronic Letters on Computer Vision and Image Analysis, vol. 7, no. 4, pp. 13-31, 2008.

[33] R. Harmouche, F. Cheriet, H. Labelle, and J. Dansereau, "3D Registration of MR and X-ray Spine Images Using an Articulated Model," Computerized Medical Imaging and Graphics, vol. 36, no. 5, pp. 410-418, 2012.

[34] R. Harmouche, F. Cheriet, H. Labelle, and J. Danserau, "Multimodal image registration of the scoliotic torso for surgical planning," BMC Medical Imaging, vol. 13, no. 1, 2013.

[35] C.-E. Aubin, H. Labelle, and O. Ciolofan, "Variability of spinal instrumentation configurations in adolescent idiopathic scoliosis," European Spine Journal, vol. 16, no. 1, pp. 57-64, 2007

[36] R. Buchanan, J.G. Birch, A.A. Morton, and R.H. Browne, "Do you see what I see? Looking at scoliosis surgical outcomes through orthopedists' eyes," Spine, vol. 28, no. 24, pp. 2700 $2705,2003$.

[37] O. Dionne, K.C. Assi, S. Grenier, H. Labelle, F. Guibault, and F. Cheriet, "Simulation of the postoperative trunk appearance in scoliosis surgery," in Proc. 9th IEEE International Symposium on Biomedical Imaging, Barcelona, May 2012. 